Yukawa Institute Kyoto

DPSU-07-2

YITP-07-39

June 2007

\title{
Interpolation of SUSY quantum mechanics
}

\author{
Satoru Odake ${ }^{a}$, Yamaç Pehlivan $^{b}$ and Ryu Sasaki ${ }^{c}$ \\ ${ }^{a}$ Department of Physics, Shinshu University, \\ Matsumoto 390-8621, Japan \\ ${ }^{b}$ Department of Physics, University of Wisconsin-Madison, \\ 1150 University Avenue, Madison WI 53706, USA \\ ${ }^{c}$ Yukawa Institute for Theoretical Physics, \\ Kyoto University, Kyoto 606-8502, Japan
}

\begin{abstract}
Interpolation of two adjacent Hamiltonians in SUSY quantum mechanics $\mathcal{H}_{s} \stackrel{\text { def }}{=}$ $(1-s) \mathcal{A}^{\dagger} \mathcal{A}+s \mathcal{A} \mathcal{A}^{\dagger}, 0 \leq s \leq 1$ is discussed together with related operators. For a wide variety of shape-invariant degree one quantum mechanics and their 'discrete' counterparts, the interpolation Hamiltonian is also shape-invariant, that is it takes the same form as the original Hamiltonian with shifted coupling constant(s).
\end{abstract}

\section{Introduction}

The factorisation method [1] or the so-called super-symmetric (SUSY) quantum mechanics [2, 3] is a well-established tool for investigating degree one quantum mechanics including their 'discrete' counterparts [4]. In its essence the SUSY quantum mechanics asserts that a factorised Hamiltonian $\mathcal{H} \stackrel{\text { def }}{=} \mathcal{A}^{\dagger} \mathcal{A}$ and its reversed order (SUSY-partner) Hamiltonian $\mathcal{H}_{r} \stackrel{\text { def }}{=} \mathcal{A} \mathcal{A}^{\dagger}$ are iso-spectral except for the ground state. Let us denote by $\phi_{n}$ the eigenfunction of $\mathcal{H}$ :

$$
\mathcal{H} \phi_{n}=\mathcal{E}_{n} \phi_{n}, \quad n=0,1,2, \ldots \quad \mathcal{E}_{0}<\mathcal{E}_{1}<\cdots
$$

Then $\tilde{\phi}_{n} \stackrel{\text { def }}{=} \mathcal{A} \phi_{n}$ is also an eigenfunction of $\mathcal{H}_{r}$ with the same eigenvalue

$$
\mathcal{H}_{r} \tilde{\phi}_{n}=\mathcal{E}_{n} \tilde{\phi}_{n}, \quad n=1,2, \ldots,
$$


except for $\phi_{0}$, which is annihilated by $\mathcal{A}$

$$
\mathcal{A} \phi_{0}=0 \quad\left(\Rightarrow \mathcal{H} \phi_{0}=0, \quad \mathcal{E}_{0}=0\right)
$$

In various contexts of quantum physics, one encounters quite often a situation [5] in which an interpolation of the two super-symmetric partner Hamiltonians

$$
\mathcal{H}_{s} \stackrel{\text { def }}{=}(1-s) \mathcal{A}^{\dagger} \mathcal{A}+s \mathcal{A} \mathcal{A}^{\dagger}, \quad 0 \leq s \leq 1
$$

or an operator closely related with it, plays an important role. For a wide class of shapeinvariant Hamiltonians [4, 6, 7], we show in this paper that the interpolation also retains shape-invariance. That is the interpolating Hamiltonian (1.4) has the same form as the original Hamiltonian with shifted coupling constant(s) and a shifted ground state energy.

This paper is organised as follows. In section two the basic facts and notation of shapeinvariant quantum mechanics are recapitulated. In section three the assertion of the shapeinvariant interpolation is demonstrated for various examples of shape-invariant potentials in ordinary quantum mechanical systems [3, 6, 7]. They have the classical orthogonal polynomials, the Hermite, Laguerre and Jacobi polynomials as a part of the eigenfunctions. In section four, we demonstrate the assertion, in a slightly different form, for various 'discrete' quantum mechanical systems [4, 7] which have the Askey-Wilson, Wilson, continuous dual Hahn, continuous Hahn and Meixner-Pollaczek polynomials [8, 9] as a part of the eigenfunctions. These polynomials belong to the Askey scheme of hypergeometric orthogonal polynomials and they are the deformations of the Jacobi, Laguerre and Hermite polynomials. In section five we apply the method presented in the previous section to the ordinary quantum mechanical systems. This gives another type of interpolation of the ordinary SUSY quantum mechanics. The final section is for a summary and comments.

\section{Shape-Invariant Quantum Mechanics}

Here we discuss only the degree one quantum mechanics. A shape-invariant [6] quantum mechanical system consists of a series of isospectral (and factorised) Hamiltonians $\{\mathcal{H}(\boldsymbol{\lambda})\}$ parametrised by (a set of) parameters $\boldsymbol{\lambda}=\left(\lambda_{1}, \lambda_{2}, \cdots\right)$ :

$$
\mathcal{H}(\boldsymbol{\lambda}) \stackrel{\text { def }}{=} \mathcal{A}(\boldsymbol{\lambda})^{\dagger} \mathcal{A}(\boldsymbol{\lambda}), \quad \mathcal{H}(\boldsymbol{\lambda}) \phi_{n}(x ; \boldsymbol{\lambda})=\mathcal{E}_{n}(\boldsymbol{\lambda}) \phi_{n}(x ; \boldsymbol{\lambda}), \quad n=0,1,2, \ldots
$$


Shape invariance simply means that the reversed order Hamiltonian

$$
\mathcal{H}_{r}(\boldsymbol{\lambda}) \stackrel{\text { def }}{=} \mathcal{A}(\boldsymbol{\lambda}) \mathcal{A}(\boldsymbol{\lambda})^{\dagger}
$$

takes the same form (shape) as the original Hamiltonian with 'shifted' parameters:

$$
\mathcal{A}(\boldsymbol{\lambda}) \mathcal{A}(\boldsymbol{\lambda})^{\dagger}=\mathcal{A}(\boldsymbol{\lambda}+\boldsymbol{\delta})^{\dagger} \mathcal{A}(\boldsymbol{\lambda}+\boldsymbol{\delta})+\mathcal{E}_{1}(\boldsymbol{\lambda}),
$$

where $\boldsymbol{\delta}$ is the shift of the parameter and $\mathcal{E}_{1}(\boldsymbol{\lambda})$ is the increase of the ground state energy. Note that the entire energy spectrum is determined by shape-invariance, since $\mathcal{E}_{n+1}(\boldsymbol{\lambda})=\mathcal{E}_{n}(\boldsymbol{\lambda})+$ $\mathcal{E}_{1}(\boldsymbol{\lambda}+n \boldsymbol{\delta})$, which implies $\left\{\mathcal{E}_{n}\right\}$ are determined in terms of $\mathcal{E}_{1}, \mathcal{E}_{n}(\boldsymbol{\lambda})=\sum_{k=0}^{n-1} \mathcal{E}_{1}(\boldsymbol{\lambda}+k \boldsymbol{\delta})$ [4, 7].

With this notation, our assertion goes as follows:

$$
\mathcal{H}_{s} \stackrel{\text { def }}{=}(1-s) \mathcal{H}+s \mathcal{H}_{r}=\mathcal{H}\left(\boldsymbol{\lambda}^{\prime}\right)+\Delta \mathcal{E}(\boldsymbol{\lambda}, s),
$$

in which the new coupling constants $\boldsymbol{\lambda}^{\prime}$ satisfy the boundary condition

$$
\text { for } s=0: \boldsymbol{\lambda}^{\prime}=\boldsymbol{\lambda}, \quad \text { and for } s=1: \boldsymbol{\lambda}^{\prime}=\boldsymbol{\lambda}+\boldsymbol{\delta} \text {. }
$$

The constant part $\Delta \mathcal{E}(\boldsymbol{\lambda}, s)$ is the shift of the ground state energy. It also satisfies the boundary condition

$$
\text { for } s=0: \Delta \mathcal{E}(\boldsymbol{\lambda}, 0)=0, \quad \text { and for } s=1: \Delta \mathcal{E}(\boldsymbol{\lambda}, 1)=\mathcal{E}_{1}(\boldsymbol{\lambda}) \text {. }
$$

Here $\mathcal{E}_{1}(\boldsymbol{\lambda})$ is the last term in (2.3).

If our assertion (2.4) holds, the eigenfunctions and eigenvalues of $\mathcal{H}_{s}$ are given by

$$
\mathcal{H}_{s} \phi_{n}\left(x ; \boldsymbol{\lambda}^{\prime}\right)=\left(\mathcal{E}_{n}\left(\boldsymbol{\lambda}^{\prime}\right)+\Delta \mathcal{E}(\boldsymbol{\lambda}, s)\right) \phi_{n}\left(x ; \boldsymbol{\lambda}^{\prime}\right), \quad n=0,1,2, \ldots
$$

Note that $\boldsymbol{\lambda}^{\prime}$ depend on $s$.

\section{Ordinary Quantum Mechanics}

The ordinary quantum mechanics is quite conveniently described by the prepotential $W(x ; \boldsymbol{\lambda})$ $\in \mathbb{R}$ which parametrises the ground state wavefunction

$$
\phi_{0}(x ; \boldsymbol{\lambda}) \propto e^{W(x ; \boldsymbol{\lambda})} .
$$


This is possible because the ground state wavefunction does not have nodes and can be chosen real. Then the factorised Hamiltonian reads

$$
\begin{aligned}
& \mathcal{H}=\mathcal{A}(\boldsymbol{\lambda})^{\dagger} \mathcal{A}(\boldsymbol{\lambda})=-\frac{d^{2}}{d x^{2}}+\left(\frac{d W(x ; \boldsymbol{\lambda})}{d x}\right)^{2}+\frac{d^{2} W(x ; \boldsymbol{\lambda})}{d x^{2}}, \\
& \mathcal{A}(\boldsymbol{\lambda}) \stackrel{\text { def }}{=}-\frac{d}{d x}+\frac{d W(x ; \boldsymbol{\lambda})}{d x}, \quad \mathcal{A}(\boldsymbol{\lambda})^{\dagger}=\frac{d}{d x}+\frac{d W(x ; \boldsymbol{\lambda})}{d x} .
\end{aligned}
$$

Here we have adopted the unit system $m=\hbar=1$ and the overall normalisation $\mathcal{H}=$ $-\frac{d^{2}}{d x^{2}}+\cdots$. Obviously the ground state is annihilated by the operator $\mathcal{A}$

$$
\mathcal{A}(\boldsymbol{\lambda}) \phi_{0}(x ; \boldsymbol{\lambda})=0 \quad\left(\Rightarrow \mathcal{H} \phi_{0}(x ; \boldsymbol{\lambda})=0, \quad \mathcal{E}_{0}(\boldsymbol{\lambda})=0\right)
$$

We assume the shape-invariance (2.3). In this form the interpolating Hamiltonian $\mathcal{H}_{s}$ reads simply

$$
\mathcal{H}_{s}=-\frac{d^{2}}{d x^{2}}+\left(\frac{d W(x ; \boldsymbol{\lambda})}{d x}\right)^{2}+(1-2 s) \frac{d^{2} W(x ; \boldsymbol{\lambda})}{d x^{2}}=\mathcal{H}-2 s \frac{d^{2} W(x ; \boldsymbol{\lambda})}{d x^{2}}
$$

Let us consider the known typical examples of shape-invariant potentials. All of them satisfy our assertion (2.4).

\section{1 harmonic oscillator: a trivial example}

The harmonic oscillator is a trivial example with $W(x ; \omega)=-\omega x^{2} / 2$. This simply leads to the same Hamiltonian with the shifted ground state energy

$$
\mathcal{H}_{s}=\mathcal{H}+\Delta \mathcal{E}(\omega, s), \quad \Delta \mathcal{E}(\omega, s)=2 s \omega
$$

\section{2 harmonic oscillator with a centrifugal barrier potential}

This is the one-body case of the Calogero model [10] with

$$
\begin{aligned}
& \boldsymbol{\lambda}=(\omega, g), \quad \boldsymbol{\delta}=(0,1), \quad \mathcal{E}_{n}(\omega, g)=4 n, \quad n=0,1,2, \ldots, \\
& W(x ; \omega, g)=-\omega x^{2} / 2+g \log x, \quad \omega>0, \quad g \geq 1 / 2, \quad x>0, \\
& \mathcal{A}(\omega, g)=-\frac{d}{d x}-\omega x+\frac{g}{x}, \quad \mathcal{A}(\omega, g)^{\dagger}=\frac{d}{d x}-\omega x+\frac{g}{x} .
\end{aligned}
$$

The original and the interpolating Hamiltonians read

$$
\begin{aligned}
\mathcal{H}(\omega, g) & =-\frac{d^{2}}{d x^{2}}+\omega^{2} x^{2}+\frac{g(g-1)}{x^{2}}-\omega(1+2 g), \\
\mathcal{H}_{s} & =-\frac{d^{2}}{d x^{2}}+\omega^{2} x^{2}+\frac{g(g+2 s-1)}{x^{2}}-\omega(1+2 g-2 s) .
\end{aligned}
$$


It is obvious that the interpolating Hamiltonian $\mathcal{H}_{s}$ has the same form as the old (3.10) with the coupling constant $g$ replaced by $g^{\prime}$ :

$$
g^{\prime}=(1+\sqrt{1+4 g(g+2 s-1)}) / 2
$$

together with the shift of the ground state energy $\Delta \mathcal{E}(\omega, g, s)=2 \omega\left(s+g^{\prime}-g\right)$.

\section{$3.31 / \sin ^{2} x$ potential or symmetric Pöschl-Teller potential}

This is the one-body case of the well-known Sutherland model [11. The prepotential, the Hamiltonian and other data are:

$$
\begin{aligned}
& \boldsymbol{\lambda}=g, \quad \boldsymbol{\delta}=1, \quad \mathcal{E}_{n}(g)=n(n+2 g), \quad n=0,1,2, \ldots, \\
& W(x ; g)=g \log \sin x, \quad g \geq 1 / 2, \quad 0<x<\pi, \\
& \mathcal{A}(g)=-\frac{d}{d x}+g \cot x, \quad \mathcal{A}(g)^{\dagger}=\frac{d}{d x}+g \cot x, \\
& \mathcal{H}=-\frac{d^{2}}{d x^{2}}+\frac{g(g-1)}{\sin ^{2} x}-g^{2} .
\end{aligned}
$$

The interpolating Hamiltonian reads simply

$$
\mathcal{H}_{s}=-\frac{d^{2}}{d x^{2}}+\frac{g(g+2 s-1)}{\sin ^{2} x}-g^{2} .
$$

It is obvious that the interpolating Hamiltonian $\mathcal{H}_{s}$ has the same form as the old (3.16) with the coupling constant $g$ replaced by $g^{\prime}$ as in the previous case (3.12) together with the shift of the ground state energy $\Delta \mathcal{E}(g, s)=g^{\prime 2}-g^{2}$.

\section{4 soliton potential or the symmetric Rosen-Morse potential}

As is well-known $-g(g+1) / \cosh ^{2} x$ potential is reflectionless for integer coupling constant

$g$, corresponding to the $\mathrm{KdV}$ soliton. It has a finite number $1+[g]^{\prime}$ (the greatest integer not equal or exceeding $g$ ) of bound states:

$$
\begin{aligned}
& \boldsymbol{\lambda}=g, \quad \boldsymbol{\delta}=-1, \quad \mathcal{E}_{n}(g)=n(2 g-n), \quad n=0,1, \ldots,[g]^{\prime}, \\
& W(x ; g)=-g \log \cosh x, \quad g>0, \quad-\infty<x<\infty \\
& \mathcal{A}(g)=-\frac{d}{d x}-g \tanh x, \quad \mathcal{A}(g)^{\dagger}=\frac{d}{d x}-g \tanh x, \\
& \mathcal{H}=-\frac{d^{2}}{d x^{2}}-\frac{g(g+1)}{\cosh ^{2} x}+g^{2} .
\end{aligned}
$$


The one-parameter Hamiltonian reads simply

$$
\mathcal{H}_{s}=-\frac{d^{2}}{d x^{2}}-\frac{g(g-2 s+1)}{\cosh ^{2} x}+g^{2} .
$$

It is obvious that the new Hamiltonian $\mathcal{H}_{s}$ has the same form as the old (3.21) with the coupling constant $g$ replaced by $g^{\prime \prime}$ (for $g>2 s-1$ case):

$$
g^{\prime \prime}=(-1+\sqrt{1+4 g(g+1-2 s)}) / 2
$$

together with the shift of the ground state energy $\Delta \mathcal{E}(g, s)=g^{2}-g^{\prime 2}$.

\subsection{Morse potential}

The prepotential, the Hamiltonian and other data of the Morse potential read

$$
\begin{aligned}
& \boldsymbol{\lambda}=(g, \mu), \quad \boldsymbol{\delta}=(-1,0), \quad \mathcal{E}_{n}(g, \mu)=n(2 g-n), \quad n=0,1, \ldots,[g]^{\prime}, \\
& W(x ; g, \mu)=g x-\mu e^{x}, \quad g, \mu>0, \quad-\infty<x<\infty \\
& \mathcal{A}(g, \mu)=-\frac{d}{d x}+g-\mu e^{x}, \quad \mathcal{A}(g, \mu)^{\dagger}=\frac{d}{d x}+g-\mu e^{x}, \\
& \mathcal{H}=-\frac{d^{2}}{d x^{2}}+\mu^{2} e^{2 x}-\mu(2 g+1) e^{x}+g^{2} .
\end{aligned}
$$

The interpolating Hamiltonian reads simply

$$
\mathcal{H}_{s}=-\frac{d^{2}}{d x^{2}}+\mu^{2} e^{2 x}-\mu(2 g-2 s+1) e^{x}+g^{2} .
$$

This has the same form as the old one with the coupling constant $g$ replaced by $g^{\prime}$ (for $g>s$ case):

$$
g^{\prime}=g-s .
$$

The shift of the ground state energy is $\Delta \mathcal{E}(g, \mu, s)=g^{2}-g^{\prime 2}$.

\section{6 hyperbolic symmetric top}

This system is obtained by the interchange $\sinh \leftrightarrow$ cosh from the hyperbolic analog of the symmetric top problem:

$$
\begin{aligned}
& \boldsymbol{\lambda}=(g, \mu), \quad \boldsymbol{\delta}=(-1,0), \quad \mathcal{E}_{n}(g, \mu)=n(2 g-n), \quad n=0,1, \ldots,[g]^{\prime}, \\
& W(x ; g, \mu)=-g \log \cosh x-\mu \arctan \sinh x, \quad g, \mu>0, \quad-\infty<x<\infty, \\
& \mathcal{A}(g, \mu)=-\frac{d}{d x}-g \tanh x-\frac{\mu}{\cosh x}, \quad \mathcal{A}(g, \mu)^{\dagger}=\frac{d}{d x}-g \tanh x-\frac{\mu}{\cosh x}, \\
& \mathcal{H}=-\frac{d^{2}}{d x^{2}}+\frac{\mu^{2}-g(g+1)+\mu(2 g+1) \sinh x}{\cosh ^{2} x}+g^{2} .
\end{aligned}
$$


The interpolating Hamiltonian reads simply

$$
\mathcal{H}_{s}=-\frac{d^{2}}{d x^{2}}+\frac{\mu^{2}-g(g-2 s+1)+\mu(2 g-2 s+1) \sinh x}{\cosh ^{2} x}+g^{2} .
$$

This has the same form as the old one with the coupling constants $(g, \mu)$ replaced by $\left(g^{\prime}, \mu^{\prime}\right)$, which are determined by

$$
\mu^{\prime 2}-g^{\prime}\left(g^{\prime}+1\right)=\mu^{2}-g(g-2 s+1), \quad \mu^{\prime}\left(2 g^{\prime}+1\right)=\mu(2 g-2 s+1) .
$$

The shift of the ground state energy is $\Delta \mathcal{E}(g, \mu, s)=g^{2}-g^{\prime 2}$. For the boundary values $s=0$ and $s=1,\left(g^{\prime}, \mu^{\prime}\right)=(g-s, \mu)$ give a solution of (3.35) $)$.

\section{7 other examples}

There are several more examples of shape-invariant quantum mechanics [3]. But the situation is the same as either the symmetric Pöschl-Teller or the symmetric Rosen-Morse potential case, with the change of the coupling constant (3.12), (3.23). We present some of them briefly.

\subsubsection{Pöschl-Teller potential}

This is the one-body case of the Sutherland model of $B C$ type:

$$
\begin{aligned}
& \boldsymbol{\lambda}=(g, h), \quad \boldsymbol{\delta}=(1,1), \quad \mathcal{E}_{n}(g, h)=4 n(n+g+h), \quad n=0,1,2, \ldots, \\
& W(x ; g, h)=g \log \sin x+h \log \cos x, \quad g, h \geq 1 / 2, \quad 0<x<\pi / 2, \\
& \mathcal{H}=-\frac{d^{2}}{d x^{2}}+\frac{g(g-1)}{\sin ^{2} x}+\frac{h(h-1)}{\cos ^{2} x}-(g+h)^{2}, \\
& \mathcal{H}_{s}=-\frac{d^{2}}{d x^{2}}+\frac{g(g+2 s-1)}{\sin ^{2} x}+\frac{h(h+2 s-1)}{\cos ^{2} x}-(g+h)^{2} .
\end{aligned}
$$

The coupling constant $g$ is replaced by $g^{\prime}$ as (3.12) and $h$ by $h^{\prime}$ similarly. The shift of the ground state energy is $\Delta \mathcal{E}(g, h, s)=\left(g^{\prime}+h^{\prime}\right)^{2}-(g+h)^{2}$.

\subsubsection{Rosen-Morse potential}

The prepotential, the Hamiltonian, etc. are:

$$
\begin{aligned}
& \boldsymbol{\lambda}=(g, \mu), \quad \boldsymbol{\delta}=(-1,0) \\
& \mathcal{E}_{n}(g, \mu)=\frac{\mu^{2}}{g^{2}}+g^{2}-\left(\frac{\mu^{2}}{(g-n)^{2}}+(g-n)^{2}\right), \quad n=0,1, \ldots,[g-\sqrt{|\mu|}]^{\prime},
\end{aligned}
$$




$$
\begin{aligned}
& W(x ; g, \mu)=-\frac{\mu}{g} x-g \log \cosh x, \quad g>0, \quad-g^{2}<\mu<g^{2}, \quad-\infty<x<\infty, \\
& \mathcal{H}=-\frac{d^{2}}{d x^{2}}+2 \mu \tanh x-\frac{g(g+1)}{\cosh ^{2} x}+\frac{\mu^{2}}{g^{2}}+g^{2}, \\
& \mathcal{H}_{s}=-\frac{d^{2}}{d x^{2}}+2 \mu \tanh x-\frac{g(g-2 s+1)}{\cosh ^{2} x}+\frac{\mu^{2}}{g^{2}}+g^{2} .
\end{aligned}
$$

The coupling constant $g$ is replaced by $g^{\prime \prime}$ as (3.23) (for $g>2 s-1$ case) but $\mu$ is unchanged. The shift of the ground state energy is $\Delta \mathcal{E}(g, \mu, s)=\mu^{2} / g^{2}+g^{2}-\left(\mu^{2} / g^{\prime \prime 2}+g^{\prime \prime 2}\right)$.

\subsubsection{Coulomb potential with the centrifugal barrier}

The prepotential, the Hamiltonian, etc. are:

$$
\begin{aligned}
& \boldsymbol{\lambda}=(g, \mu), \quad \boldsymbol{\delta}=(1,0), \quad \mathcal{E}_{n}(g, \mu)=\frac{\mu^{2}}{g^{2}}-\frac{\mu^{2}}{(g+n)^{2}}, \quad n=0,1,2, \ldots, \\
& W(x ; g, \mu)=-\frac{\mu}{g} x+g \log x, \quad g \geq \frac{1}{2}, \quad \mu>0, \quad x>0 \\
& \mathcal{H}=-\frac{d^{2}}{d x^{2}}-\frac{2 \mu}{x}+\frac{g(g-1)}{x^{2}}+\frac{\mu^{2}}{g^{2}} \\
& \mathcal{H}_{s}=-\frac{d^{2}}{d x^{2}}-\frac{2 \mu}{x}+\frac{g(g+2 s-1)}{x^{2}}+\frac{\mu^{2}}{g^{2}} .
\end{aligned}
$$

The coupling constant $g$ is replaced by $g^{\prime}$ as (3.12) but $\mu$ is unchanged. The shift of the ground state energy is $\Delta \mathcal{E}(g, \mu, s)=\mu^{2} / g^{2}-\mu^{2} / g^{\prime 2}$.

\section{Discrete Quantum Mechanics}

The corresponding result for discrete quantum mechanics takes a slightly different form. Let us start with the brief introduction of the general setting of the discrete quantum mechanics. For more details we refer to [4, 7, 12].

The Hamiltonian of discrete quantum mechanics has a generic form

$$
\mathcal{H}=\sqrt{V(x ; \boldsymbol{\lambda})} e^{-i \partial_{x}} \sqrt{V(x ; \boldsymbol{\lambda})^{*}}+\sqrt{V(x ; \boldsymbol{\lambda})^{*}} e^{i \partial_{x}} \sqrt{V(x ; \boldsymbol{\lambda})}-V(x ; \boldsymbol{\lambda})-V(x ; \boldsymbol{\lambda})^{*},
$$

for various potential functions $V(x ; \boldsymbol{\lambda})$, which are in general complex. The corresponding Schrödinger equation is a difference equation in stead of a differential equation. It is also factorised, $\mathcal{H}=\mathcal{A}^{\dagger} \mathcal{A}$, with

$$
\begin{aligned}
\mathcal{A} & =\mathcal{A}(\boldsymbol{\lambda}) \stackrel{\text { def }}{=}-i\left(e^{-\frac{i}{2} \partial_{x}} \sqrt{V(x ; \boldsymbol{\lambda})^{*}}-e^{\frac{i}{2} \partial_{x}} \sqrt{V(x ; \boldsymbol{\lambda})}\right), \\
\mathcal{A}^{\dagger} & =\mathcal{A}(\boldsymbol{\lambda})^{\dagger}=i\left(\sqrt{V(x ; \boldsymbol{\lambda})} e^{-\frac{i}{2} \partial_{x}}-\sqrt{V(x ; \boldsymbol{\lambda})^{*}} e^{\frac{i}{2} \partial_{x}}\right) .
\end{aligned}
$$


As in the ordinary quantum mechanics cases, the ground state wavefunction $\phi_{0}$ is annihilated by the operator $\mathcal{A}$ :

$$
\mathcal{A}(\boldsymbol{\lambda}) \phi_{0}(x ; \boldsymbol{\lambda})=0 \quad\left(\Rightarrow \mathcal{H} \phi_{0}(x ; \boldsymbol{\lambda})=0, \quad \mathcal{E}_{0}(\boldsymbol{\lambda})=0\right)
$$

We introduce a similarity transformed Hamiltonian $\tilde{\mathcal{H}}(\boldsymbol{\lambda})$ in terms of the ground state wavefunction $\phi_{0}=\phi_{0}(x ; \boldsymbol{\lambda})$ :

$$
\tilde{\mathcal{H}} \stackrel{\text { def }}{=} \phi_{0}^{-1} \circ \mathcal{H} \circ \phi_{0}=V(x ; \boldsymbol{\lambda}) e^{-i \partial_{x}}+V(x ; \boldsymbol{\lambda})^{*} e^{i \partial_{x}}-V(x ; \boldsymbol{\lambda})-V(x ; \boldsymbol{\lambda})^{*} .
$$

It acts on the polynomial part of the eigenfunction

$$
\begin{aligned}
& \phi_{n}(x ; \boldsymbol{\lambda}) \propto \phi_{0}(x ; \boldsymbol{\lambda}) P_{n}(\eta(x) ; \boldsymbol{\lambda}), \\
& \tilde{\mathcal{H}} P_{n}(\eta(x) ; \boldsymbol{\lambda})=\mathcal{E}_{n}(\boldsymbol{\lambda}) P_{n}(\eta(x) ; \boldsymbol{\lambda}) .
\end{aligned}
$$

Here $P_{n}(\eta ; \boldsymbol{\lambda})$ is a polynomial of degree $n$ in variable $\eta$, and $\eta(x)$ is a real function of $x$. For all the examples given in this section, the eigenfunctions have this form.

We assume that the system is shape-invariant (2.3). Next let us introduce the similarity transformation of the reversed order Hamiltonian $\mathcal{H}_{r}(\boldsymbol{\lambda})=\mathcal{A}(\boldsymbol{\lambda}) \mathcal{A}(\boldsymbol{\lambda})^{\dagger}$ :

$$
\begin{aligned}
& \tilde{\mathcal{H}}_{r} \stackrel{\text { def }}{=} \phi_{r 0}^{-1} \circ \mathcal{H}_{r} \circ \phi_{r 0} \\
& =V(x ; \boldsymbol{\lambda}+\boldsymbol{\delta}) e^{-i \partial_{x}}+V(x ; \boldsymbol{\lambda}+\boldsymbol{\delta})^{*} e^{i \partial_{x}}-V(x ; \boldsymbol{\lambda}+\boldsymbol{\delta})-V(x ; \boldsymbol{\lambda}+\boldsymbol{\delta})^{*}+\mathcal{E}_{1}(\boldsymbol{\lambda}),
\end{aligned}
$$

with respect to its ground state wavefunction $\phi_{r 0}=\phi_{r 0}(x ; \boldsymbol{\lambda})$ defined by

$$
\mathcal{A}(\boldsymbol{\lambda}+\boldsymbol{\delta}) \phi_{r 0}(x ; \boldsymbol{\lambda})=0 \quad\left(\Rightarrow \phi_{r 0}(x ; \boldsymbol{\lambda}) \propto \phi_{0}(x ; \boldsymbol{\lambda}+\boldsymbol{\delta})\right)
$$

We introduce the one parameter family of (similarity transformed) Hamiltonians:

$$
\begin{aligned}
\tilde{\mathcal{H}}_{s} & \stackrel{\text { def }}{=}(1-s) \tilde{\mathcal{H}}+s \tilde{\mathcal{H}}_{r} \\
& =V_{s}(x ; \boldsymbol{\lambda}) e^{-i \partial_{x}}+V_{s}(x ; \boldsymbol{\lambda})^{*} e^{i \partial_{x}}-V_{s}(x ; \boldsymbol{\lambda})-V_{s}(x ; \boldsymbol{\lambda})^{*}+s \mathcal{E}_{1}(\boldsymbol{\lambda}),
\end{aligned}
$$

which depends on the interpolated potential function $V_{s}(x ; \boldsymbol{\lambda})$ :

$$
V_{s}(x ; \boldsymbol{\lambda}) \stackrel{\text { def }}{=}(1-s) V(x ; \boldsymbol{\lambda})+s V(x ; \boldsymbol{\lambda}+\boldsymbol{\delta}) .
$$

Since both $\tilde{\mathcal{H}}$ and $\tilde{\mathcal{H}}_{r}$ act on the space of polynomials, their sum is meaningful and acts on the same space. 
Our assertion is that $V_{s}(x ; \boldsymbol{\lambda})$ is simply the same potential function with shifted coupling constants $\lambda^{\prime}$ :

$$
V_{s}(x ; \boldsymbol{\lambda})=V\left(x ; \boldsymbol{\lambda}^{\prime}\right)
$$

Then we have

$$
\tilde{\mathcal{H}}_{s}=\tilde{\mathcal{H}}\left(\boldsymbol{\lambda}^{\prime}\right)+\widetilde{\Delta \mathcal{E}}(\boldsymbol{\lambda}, s), \quad \widetilde{\Delta \mathcal{E}}(\boldsymbol{\lambda}, s)=s \mathcal{E}_{1}(\boldsymbol{\lambda})
$$

Recall that $\boldsymbol{\lambda}^{\prime}$ depend on $s$. The eigenfunctions and eigenvalues of $\tilde{\mathcal{H}}_{s}$ are given by

$$
\tilde{\mathcal{H}}_{s} P_{n}\left(\eta(x) ; \boldsymbol{\lambda}^{\prime}\right)=\left(\mathcal{E}_{n}\left(\boldsymbol{\lambda}^{\prime}\right)+s \mathcal{E}_{1}(\boldsymbol{\lambda})\right) P_{n}\left(\eta(x) ; \boldsymbol{\lambda}^{\prime}\right), \quad n=0,1,2, \ldots
$$

By similarity transformation inversely with respect to $\phi_{s 0}=\phi_{s 0}(x ; \boldsymbol{\lambda})$ defined by

$$
\mathcal{A}\left(\boldsymbol{\lambda}^{\prime}\right) \phi_{s 0}(x ; \boldsymbol{\lambda})=0 \quad \Longrightarrow \phi_{s 0}(x ; \boldsymbol{\lambda}) \propto \phi_{0}\left(x ; \boldsymbol{\lambda}^{\prime}\right)
$$

we introduce $\check{\mathcal{H}}_{s}$ as

$$
\begin{aligned}
\check{\mathcal{H}}_{s} & \stackrel{\text { def }}{=} \phi_{s 0} \circ \tilde{\mathcal{H}}_{s} \circ \phi_{s 0}^{-1} \\
& =(1-s) \frac{\phi_{0}\left(x ; \boldsymbol{\lambda}^{\prime}\right)}{\phi_{0}(x ; \boldsymbol{\lambda})} \circ \mathcal{H} \circ \frac{\phi_{0}(x ; \boldsymbol{\lambda})}{\phi_{0}\left(x ; \boldsymbol{\lambda}^{\prime}\right)}+s \frac{\phi_{0}\left(x ; \boldsymbol{\lambda}^{\prime}\right)}{\phi_{0}(x ; \boldsymbol{\lambda}+\boldsymbol{\delta})} \circ \mathcal{H}_{r} \circ \frac{\phi_{0}(x ; \boldsymbol{\lambda}+\boldsymbol{\delta})}{\phi_{0}\left(x ; \boldsymbol{\lambda}^{\prime}\right)} \\
& =\mathcal{H}\left(\boldsymbol{\lambda}^{\prime}\right)+s \mathcal{E}_{1}(\boldsymbol{\lambda}) .
\end{aligned}
$$

This one parameter family of Hamiltonians $\check{\mathcal{H}}_{s}$ interpolates $\mathcal{H}(s=0)$ and $\mathcal{H}_{r}(s=1)$. Its eigenfunctions and eigenvalues are given by

$$
\check{\mathcal{H}}_{s} \phi_{n}\left(x ; \boldsymbol{\lambda}^{\prime}\right)=\left(\mathcal{E}_{n}\left(\boldsymbol{\lambda}^{\prime}\right)+s \mathcal{E}_{1}(\boldsymbol{\lambda})\right) \phi_{n}\left(x ; \boldsymbol{\lambda}^{\prime}\right), \quad n=0,1,2, \ldots
$$

From this our claim follows

the interpolated (similarity transformed) Hamiltonian $\check{\mathcal{H}}_{s}\left(\tilde{\mathcal{H}}_{s}\right)(0 \leq s \leq 1)$ also describes the same shape-invariant and exactly solvable system with shifted coupling constants.

This claim can be easily verified for each known shape-invariant discrete quantum mechanics given below. We will denote them by the name of the polynomials $\left\{P_{n}(\eta)\right\}$ constituting the eigenfunctions. 


\subsection{Meixner-Pollaczek polynomial}

The Meixner-Pollaczek polynomial [4, 9, 13] is a one-parameter deformation of the Hermite polynomial. Therefore the corresponding discrete quantum mechanics is also called a deformed harmonic oscillator. Its potential function and other data are:

$$
\begin{aligned}
& \boldsymbol{\lambda}=\lambda, \quad \boldsymbol{\delta}=1 / 2, \quad \mathcal{E}_{n}(\lambda)=2 n, \quad n=0,1,2, \ldots, \\
& V(x ; \lambda)=\lambda+i x, \quad \lambda>0, \quad-\infty<x<\infty
\end{aligned}
$$

The increase of the ground state energy $\mathcal{E}_{1}(\boldsymbol{\lambda})=2$ is independent of the coupling constant as for the ordinary harmonic oscillator. It is rather trivial to verify our assertion:

$$
V_{s}(x ; \lambda)=\lambda+s / 2+i x
$$

and

$$
\lambda^{\prime}=\lambda+s / 2, \quad \widetilde{\Delta \mathcal{E}}(\lambda ; s)=2 s
$$

\section{2 continuous Hahn polynomial}

The continuous Hahn polynomial with special parameters [4, 9] is a two-parameter deformation of the Hermite polynomial. The potential function and the other data are:

$$
\begin{aligned}
& \boldsymbol{\lambda}=(a, b), \quad \boldsymbol{\delta}=(1 / 2,1 / 2), \quad \mathcal{E}_{n}(\boldsymbol{\lambda})=n(n+2 a+2 b-1), \quad n=0,1,2, \ldots, \\
& V(x ; \boldsymbol{\lambda})=(a+i x)(b+i x), \quad a, b>0, \quad-\infty<x<\infty .
\end{aligned}
$$

These lead to $\boldsymbol{\lambda}^{\prime}=\left(a^{\prime}, b^{\prime}\right)$ determined by

$$
a^{\prime}+b^{\prime}=a+b+s, \quad a^{\prime} b^{\prime}=a b+(a+b) s / 2+s / 4
$$

and

$$
\widetilde{\Delta \mathcal{E}}(\boldsymbol{\lambda} ; s)=2(a+b) s
$$

For the boundary values $s=0$ and $s=1,\left(a^{\prime}, b^{\prime}\right)=(a+s / 2, b+s / 2)$ is a solution of (4.25).

\section{3 continuous dual Hahn polynomial}

The continuous dual Hahn polynomial [4, 9] is a two-parameter deformation of the Laguerre polynomial. Therefore the corresponding quantum mechanics is a deformation of the potential $x^{2}+1 / x^{2}$, or the one body case of the Calogero model [10] discussed in $\$ 3.2$. The 
potential function and the other data are:

$$
\begin{aligned}
& \boldsymbol{\lambda}=(a, b, c), \quad \boldsymbol{\delta}=(1 / 2,1 / 2,1 / 2), \quad \mathcal{E}_{n}(\boldsymbol{\lambda})=n, \quad n=0,1,2, \ldots, \\
& V(x ; \boldsymbol{\lambda})=\frac{(a+i x)(b+i x)(c+i x)}{2 i x(2 i x+1)}, \quad a, b, c>0, \quad 0<x<\infty .
\end{aligned}
$$

As in the Calogero case, the increase of the ground state energy is independent of the coupling constants and this leads to the linear energy spectrum. The shifted parameters $\boldsymbol{\lambda}^{\prime}=\left(a^{\prime}, b^{\prime}, c^{\prime}\right)$ are determined by

$$
\begin{aligned}
a^{\prime}+b^{\prime}+c^{\prime} & =a+b+c+3 s / 2, \\
a^{\prime} b^{\prime}+b^{\prime} c^{\prime}+c^{\prime} a^{\prime} & =a b+b c+c a+(a+b+c) s+3 s / 4, \\
a^{\prime} b^{\prime} c^{\prime} & =a b c+(a b+b c+c a) s / 2+(a+b+c) s / 4+s / 8
\end{aligned}
$$

and

$$
\widetilde{\Delta \mathcal{E}}(\boldsymbol{\lambda} ; s)=s .
$$

For the boundary values $s=0$ and $s=1,\left(a^{\prime}, b^{\prime}, c^{\prime}\right)=(a+s / 2, b+s / 2, c+s / 2)$ is a solution of (4.29)-(4.31).

\subsection{Wilson polynomial}

The Wilson polynomial [4, 9] is a three-parameter deformation of the Laguerre polynomial. The potential function and the other data are:

$$
\begin{array}{ll}
\boldsymbol{\lambda}=(a, b, c, d), \quad \boldsymbol{\delta}=(1 / 2,1 / 2,1 / 2,1 / 2), & \\
\mathcal{E}_{n}(\boldsymbol{\lambda})=n(n+a+b+c+d-1), & n=0,1,2, \ldots, \\
V(x ; \boldsymbol{\lambda})=\frac{(a+i x)(b+i x)(c+i x)(d+i x)}{2 i x(2 i x+1)}, & a, b, c, d>0, \quad 0<x<\infty .
\end{array}
$$

The shifted parameters $\boldsymbol{\lambda}^{\prime}=\left(a^{\prime}, b^{\prime}, c^{\prime}, d^{\prime}\right)$ are determined by

$$
\begin{aligned}
& a^{\prime}+b^{\prime}+c^{\prime}+d^{\prime}=a+b+c+d+2 s \\
& a^{\prime} b^{\prime}+a^{\prime} c^{\prime}+a^{\prime} d^{\prime}+b^{\prime} c^{\prime}+b^{\prime} d^{\prime}+c^{\prime} d^{\prime} \\
& =a b+a c+a d+b c+b d+c d+3(a+b+c+d) s / 2+3 s / 2 \\
& a^{\prime} b^{\prime} c^{\prime}+a^{\prime} b^{\prime} d^{\prime}+a^{\prime} c^{\prime} d^{\prime}+b^{\prime} c^{\prime} d^{\prime} \\
& =a b c+a b d+a c d+b c d+(a b+a c+a d+b c+b d+c d) s+3(a+b+c+d) s / 4+s / 2 \\
& a^{\prime} b^{\prime} c^{\prime} d^{\prime}=a b c d+(a b c+a b d+a c d+b c d) s / 2+(a b+a c+a d+b c+b d+c d) s / 4 \\
& \quad+(a+b+c+d) s / 8+s / 16
\end{aligned}
$$


and

$$
\widetilde{\Delta \mathcal{E}}(\boldsymbol{\lambda} ; s)=(a+b+c+d) s .
$$

For the boundary values $s=0$ and $s=1,\left(a^{\prime}, b^{\prime}, c^{\prime}, d^{\prime}\right)=(a+s / 2, b+s / 2, c+s / 2, d+s / 2)$ is a solution of (4.36)-(4.39).

\subsection{Askey-Wilson polynomial}

The Askey-Wilson polynomial 4, 9] belongs to a different type of shape-invariant discrete quantum mechanical single particle systems. It has a multiplicative shift of parameters rather than the additive shift (2.3) discussed in the rest of this paper.

Following [4, 7, 12], we use variables $\theta, x$ and $z$, which are related as

$$
0 \leq \theta \leq \pi, \quad x=\cos \theta, \quad z=e^{i \theta} .
$$

The dynamical variable is $\theta$ and the inner product is $\langle f \mid g\rangle=\int_{0}^{\pi} d \theta f(\theta)^{*} g(\theta)$. We denote $D \stackrel{\text { def }}{=} z \frac{d}{d z}$. Then $q^{D}$ is a $q$-shift operator, $q^{D} f(z)=f(q z)$. We assume $0<q<1$. We note here that

$$
\int_{0}^{\pi} d \theta=\int_{-1}^{1} \frac{d x}{\sqrt{1-x^{2}}}, \quad-i \frac{d}{d \theta}=z \frac{d}{d z}=D
$$

The Hamiltonian is

$$
\mathcal{H}=\sqrt{V(z ; \boldsymbol{\lambda})} q^{D} \sqrt{V(z ; \boldsymbol{\lambda})^{*}}+\sqrt{V(z ; \boldsymbol{\lambda})^{*}} q^{-D} \sqrt{V(z ; \boldsymbol{\lambda})}-V(z ; \boldsymbol{\lambda})-V(z ; \boldsymbol{\lambda})^{*},
$$

which is factorized, i.e. $\mathcal{H}=\mathcal{A}^{\dagger} \mathcal{A}$, with

$$
\begin{aligned}
& \mathcal{A}=\mathcal{A}(\boldsymbol{\lambda}) \stackrel{\text { def }}{=} i\left(q^{\frac{D}{2}} \sqrt{V(z ; \boldsymbol{\lambda})^{*}}-q^{-\frac{D}{2}} \sqrt{V(z ; \boldsymbol{\lambda})}\right), \\
& \mathcal{A}^{\dagger}=\mathcal{A}(\boldsymbol{\lambda})^{\dagger}=-i\left(\sqrt{V(z ; \boldsymbol{\lambda})} q^{\frac{D}{2}}-\sqrt{V(z ; \boldsymbol{\lambda})^{*}} q^{-\frac{D}{2}}\right) .
\end{aligned}
$$

The ground state $\phi_{0}$ is the function annihilated by $\mathcal{A}$ :

$$
\mathcal{A}(\boldsymbol{\lambda}) \phi_{0}(z ; \boldsymbol{\lambda})=0 \quad\left(\Rightarrow \mathcal{H} \phi_{0}(z ; \boldsymbol{\lambda})=0, \quad \mathcal{E}_{0}(\boldsymbol{\lambda})=0\right) .
$$

The potential function and other data are

$$
\begin{aligned}
& \boldsymbol{\lambda}=(a, b, c, d), \quad-1<a, b, c, d<1, \quad a b c d<q, \\
& \mathcal{E}_{n}(\boldsymbol{\lambda})=\left(q^{-n}-1\right)\left(1-a b c d q^{n-1}\right), \quad n=0,1,2, \ldots, \\
& V(z ; \boldsymbol{\lambda})=\frac{(1-a z)(1-b z)(1-c z)(1-d z)}{\left(1-z^{2}\right)\left(1-q z^{2}\right)} .
\end{aligned}
$$


The similarity transformed Hamiltonian in terms of the ground state wavefunction $\phi_{0}=$ $\phi_{0}(z ; \boldsymbol{\lambda})$ reads

$$
\tilde{\mathcal{H}} \stackrel{\text { def }}{=} \phi_{0}^{-1} \circ \mathcal{H} \circ \phi_{0}=V(z ; \boldsymbol{\lambda}) q^{D}+V(z ; \boldsymbol{\lambda})^{*} q^{-D}-V(z ; \boldsymbol{\lambda})-V(z ; \boldsymbol{\lambda})^{*} .
$$

The shape-invariance relation reads

$$
\mathcal{A}(\boldsymbol{\lambda}) \mathcal{A}(\boldsymbol{\lambda})^{\dagger}=q^{-1} \mathcal{A}\left(q^{\frac{1}{2}} \boldsymbol{\lambda}\right)^{\dagger} \mathcal{A}\left(q^{\frac{1}{2}} \boldsymbol{\lambda}\right)+\mathcal{E}_{1}(\boldsymbol{\lambda}) .
$$

The similarity transformed reversed order Hamiltonian $\mathcal{H}_{r}(\boldsymbol{\lambda})=\mathcal{A}(\boldsymbol{\lambda}) \mathcal{A}(\boldsymbol{\lambda})^{\dagger}$, with respect to its ground state wavefunction $\phi_{r 0}=\phi_{r 0}(z ; \boldsymbol{\lambda})\left(\mathcal{A}\left(q^{\frac{1}{2}} \boldsymbol{\lambda}\right) \phi_{r 0}(z ; \boldsymbol{\lambda})=0 \Rightarrow \phi_{r 0}(z ; \boldsymbol{\lambda}) \propto\right.$ $\left.\phi_{0}\left(z ; q^{\frac{1}{2}} \boldsymbol{\lambda}\right)\right)$, reads

$$
\begin{aligned}
& \tilde{\mathcal{H}}_{r} \stackrel{\text { def }}{=} \phi_{r 0}^{-1} \circ \mathcal{H}_{r} \circ \phi_{r 0} \\
& =q^{-1}\left(V\left(z ; q^{\frac{1}{2}} \boldsymbol{\lambda}\right) q^{D}+V\left(z ; q^{\frac{1}{2}} \boldsymbol{\lambda}\right)^{*} q^{-D}-V\left(z ; q^{\frac{1}{2}} \boldsymbol{\lambda}\right)-V\left(z ; q^{\frac{1}{2}} \boldsymbol{\lambda}\right)^{*}\right)+\mathcal{E}_{1}(\boldsymbol{\lambda}) .
\end{aligned}
$$

The one parameter family of (similarity transformed) Hamiltonians is introduced as

$$
\begin{aligned}
\tilde{\mathcal{H}}_{s} & \stackrel{\text { def }}{=}(1-s) \tilde{\mathcal{H}}+s \tilde{\mathcal{H}}_{r} \\
& =V_{s}(z ; \boldsymbol{\lambda}) q^{D}+V_{s}(z ; \boldsymbol{\lambda})^{*} q^{-D}-V_{s}(z ; \boldsymbol{\lambda})-V_{s}(z ; \boldsymbol{\lambda})^{*}+s \mathcal{E}_{1}(\boldsymbol{\lambda}),
\end{aligned}
$$

which depends on the interpolated potential function $V_{s}(z ; \boldsymbol{\lambda})$ :

$$
V_{s}(z ; \boldsymbol{\lambda}) \stackrel{\text { def }}{=}(1-s) V(z ; \boldsymbol{\lambda})+s q^{-1} V\left(z ; q^{\frac{1}{2}} \boldsymbol{\lambda}\right) .
$$

Our assertion is that $V_{s}(z ; \boldsymbol{\lambda})$ is simply the same potential function with shifted coupling constants $\boldsymbol{\lambda}^{\prime}=\left(a^{\prime}, b^{\prime}, c^{\prime}, d^{\prime}\right)$ with an overall scaling factor $\alpha>0$ :

$$
V_{s}(z ; \boldsymbol{\lambda})=\alpha V\left(z ; \boldsymbol{\lambda}^{\prime}\right)
$$

Then we have

$$
\tilde{\mathcal{H}}_{s}=\alpha \tilde{\mathcal{H}}\left(\boldsymbol{\lambda}^{\prime}\right)+\widetilde{\Delta \mathcal{E}}(\boldsymbol{\lambda}, s), \quad \widetilde{\Delta \mathcal{E}}(\boldsymbol{\lambda}, s)=s \mathcal{E}_{1}(\boldsymbol{\lambda}) .
$$

We can define $\check{\mathcal{H}}_{s}$ like as (4.16). Equations like as (4.15) and (4.18) hold also. 
The shifted parameters are determined by

$$
\begin{aligned}
\alpha & =1+\left(q^{-1}-1\right) s, \\
a^{\prime}+b^{\prime}+c^{\prime}+d^{\prime} & =\frac{1+\left(q^{-1 / 2}-1\right) s}{1+\left(q^{-1}-1\right) s}(a+b+c+d), \\
a^{\prime} b^{\prime}+a^{\prime} c^{\prime}+a^{\prime} d^{\prime}+b^{\prime} c^{\prime}+b^{\prime} d^{\prime}+c^{\prime} d^{\prime} & =\frac{1}{1+\left(q^{-1}-1\right) s}(a b+a c+a d+b c+b d+c d), \\
a^{\prime} b^{\prime} c^{\prime}+a^{\prime} b^{\prime} d^{\prime}+a^{\prime} c^{\prime} d^{\prime}+b^{\prime} c^{\prime} d^{\prime} & =\frac{1-\left(1-q^{1 / 2}\right) s}{1+\left(q^{-1}-1\right) s}(a b c+a b d+a c d+b c d), \\
a^{\prime} b^{\prime} c^{\prime} d^{\prime} & =\frac{1-(1-q) s}{1+\left(q^{-1}-1\right) s} a b c d
\end{aligned}
$$

and

$$
\widetilde{\Delta \mathcal{E}}(\boldsymbol{\lambda}, s)=\left(q^{-1}-1\right)(1-a b c d) s .
$$

For the boundary values $s=0$ and $s=1, \alpha=q^{-s}$ and $\left(a^{\prime}, b^{\prime}, c^{\prime}, d^{\prime}\right)=q^{s / 2}(a, b, c, d)$ is a solution of (4.57)-(4.61).

\section{Ordinary Quantum Mechanics Revisited}

In the previous section we have considered the one parameter family of (similarity transformed) Hamiltonians (4.10), (4.16). This construction method applies also to the ordinary quantum mechanics studied in section 3 .

We introduce a similarity transformed Hamiltonian $\tilde{\mathcal{H}}$ in terms of the ground state wavefunction $\phi_{0}=\phi_{0}(x ; \boldsymbol{\lambda}) \propto e^{W(x ; \boldsymbol{\lambda})}$ :

$$
\tilde{\mathcal{H}} \stackrel{\text { def }}{=} \phi_{0}^{-1} \circ \mathcal{H} \circ \phi_{0}=-\frac{d^{2}}{d x^{2}}-2 \frac{d W(x ; \boldsymbol{\lambda})}{d x} \frac{d}{d x} .
$$

Next let us introduce the similarity transformation of the reversed order Hamiltonian (2.2), (2.3) with respect to its ground state wavefunction $\phi_{r 0}=\phi_{r 0}(x ; \boldsymbol{\lambda}) \propto e^{W(x ; \boldsymbol{\lambda}+\boldsymbol{\delta})}$ :

$$
\tilde{\mathcal{H}}_{r} \stackrel{\text { def }}{=} \phi_{r 0}^{-1} \circ \mathcal{H}_{r} \circ \phi_{r 0}=-\frac{d^{2}}{d x^{2}}-2 \frac{d W(x ; \boldsymbol{\lambda}+\boldsymbol{\delta})}{d x} \frac{d}{d x}+\mathcal{E}_{1}(\boldsymbol{\lambda}) .
$$

The one parameter family of similarity transformed Hamiltonians is introduced as (4.10):

$$
\tilde{\mathcal{H}}_{s} \stackrel{\text { def }}{=}(1-s) \tilde{\mathcal{H}}+s \tilde{\mathcal{H}}_{r}=-\frac{d^{2}}{d x^{2}}-2 \frac{d W_{s}(x ; \boldsymbol{\lambda})}{d x} \frac{d}{d x}+s \mathcal{E}_{1}(\boldsymbol{\lambda})
$$

where the interpolated prepotential $W_{s}(x ; \boldsymbol{\lambda})$ is given by

$$
W_{s}(x ; \boldsymbol{\lambda}) \stackrel{\text { def }}{=}(1-s) W(x ; \boldsymbol{\lambda})+s W(x ; \boldsymbol{\lambda}+\boldsymbol{\delta}) .
$$


We do not assume (4.6) in general.

Our assertion is that $W_{s}(x ; \boldsymbol{\lambda})$ is simply the same prepotential with shifted coupling constants $\boldsymbol{\lambda}^{\prime}$ :

$$
W_{s}(x ; \boldsymbol{\lambda})=W\left(x ; \boldsymbol{\lambda}^{\prime}\right) .
$$

Then we have

$$
\tilde{\mathcal{H}}_{s}=\tilde{\mathcal{H}}\left(\boldsymbol{\lambda}^{\prime}\right)+\widetilde{\Delta \mathcal{E}}(\boldsymbol{\lambda}, s), \quad \widetilde{\Delta \mathcal{E}}(\boldsymbol{\lambda}, s)=s \mathcal{E}_{1}(\boldsymbol{\lambda}) .
$$

Note that $\boldsymbol{\lambda}^{\prime}$ depend on $s$. As in (4.16), we define inversely similarity transformed Hamiltonian with respect to $\phi_{s 0}=\phi_{s 0}(x ; \boldsymbol{\lambda}) \propto e^{W_{s}(x ; \boldsymbol{\lambda})}=e^{W\left(x ; \boldsymbol{\lambda}^{\prime}\right)}$ :

$$
\begin{aligned}
\check{\mathcal{H}}_{s} & \stackrel{\text { def }}{=} \phi_{s 0} \circ \tilde{\mathcal{H}}_{s} \circ \phi_{s 0}^{-1} \\
& =(1-s) \frac{\phi_{0}\left(x ; \boldsymbol{\lambda}^{\prime}\right)}{\phi_{0}(x ; \boldsymbol{\lambda})} \circ \mathcal{H} \circ \frac{\phi_{0}(x ; \boldsymbol{\lambda})}{\phi_{0}\left(x ; \boldsymbol{\lambda}^{\prime}\right)}+s \frac{\phi_{0}\left(x ; \boldsymbol{\lambda}^{\prime}\right)}{\phi_{0}(x ; \boldsymbol{\lambda}+\boldsymbol{\delta})} \circ \mathcal{H}_{r} \circ \frac{\phi_{0}(x ; \boldsymbol{\lambda}+\boldsymbol{\delta})}{\phi_{0}\left(x ; \boldsymbol{\lambda}^{\prime}\right)} \\
& =\mathcal{H}\left(\boldsymbol{\lambda}^{\prime}\right)+s \mathcal{E}_{1}(\boldsymbol{\lambda}) .
\end{aligned}
$$

This one parameter family of Hamiltonians $\check{\mathcal{H}}_{s}$ interpolates $\mathcal{H}(s=0)$ and $\mathcal{H}_{r}(s=1)$, and its eigenfunctions and eigenvalues have the same form as those in (4.18). This interpolation is different from the one studied in section 3 ,

For the quantum mechanical systems studied in section 3, we give a list of $\boldsymbol{\lambda}^{\prime}$ :

$$
\begin{array}{ll}
\$ 3.1 \quad: \quad \omega^{\prime}=\omega, \\
\$ 3.2 \quad: \quad \omega^{\prime}=\omega, \quad g^{\prime}=g+s, \\
\$ 3.3 \quad: \quad g^{\prime}=g+s, \\
\$ 3.4 \quad: \quad g^{\prime}=g-s, \\
\$ 3.5 \quad: \quad g^{\prime}=g-s, \quad \mu^{\prime}=\mu, \\
\$ 3.6 \quad: \quad g^{\prime}=g-s, \quad \mu^{\prime}=\mu, \\
\$ 3.7 .1: & g^{\prime}=g+s, \quad h^{\prime}=h+s, \\
\$ 3.7 .2: & g^{\prime}=g-s, \quad \mu^{\prime}=\frac{(g-s)(g-1+s)}{g(g-1)} \mu, \\
&
\end{array}
$$

\section{Summary and Comments}

Interpolation of the SUSY-partner Hamiltonians are discussed for various shape-invariant quantum mechanics including the discrete ones. For ordinary quantum mechanics we present 
two kinds of interpolation, $\mathcal{H}_{s}$ and $\check{\mathcal{H}}_{s}\left(\right.$ or $\left.\tilde{\mathcal{H}}_{s}\right)$. The shape-invariance is inherited by the interpolating Hamiltonian with shifted coupling constants. The interpolation works at the corresponding eigenfunctions level. For the quantum systems whose eigenfunctions have the form (4.6), the annihilation-creation operators can be constructed [12]. These annihilationcreation operators are also interpolated. The eigenfunctions of annihilation operators are interpreted as coherent states. Therefore these coherent states are also interpolated.

It would be interesting if a similar interpolation idea works for a wider class of quantum mechanics [14] which do not have shape-invariance.

\section{Acknowledgements}

This work is supported in part by Grants-in-Aid for Scientific Research from the Ministry of Education, Culture, Sports, Science and Technology, No.18340061 and No.19540179, in part by the U.S. National Science Foundation Grant No. PHY-0555231 at the University of Wisconsin and in part by the University of Wisconsin Research Committee with funds granted by the Wisconsin Alumni Research Foundation.

\section{References}

[1] M. M. Crum, "Associated Sturm-Liouville systems," Quart. J. Math. Oxford Ser. (2) 6 (1955) 121-127, arXiv:physics/9908019; L. Infeld and T. E. Hull, "The factorization method," Rev. Mod. Phys. 23 (1951) 21-68.

[2] E. Witten, "Dynamical breaking of supersymmetry," Nucl. Phys. B188 (1981) 513-554.

[3] See, for example: F. Cooper, A. Khare and U. Sukhatme, "Supersymmetry and quantum mechanics," Phys. Rept. 251 (1995) 267-385, arXiv:hep-th/9405029.

[4] S. Odake and R. Sasaki, "Shape Invariant Potentials in 'Discrete' Quantum Mechanics," J. Nonlinear Math. Phys. 12 Suppl. 1 (2005) 507-521, arXiv:hep-th/0410102; "Equilibrium Positions, Shape Invariance and Askey-Wilson Polynomials," J. Math. Phys. 46 (2005) 063513 (10 pages), arXiv:hep-th/0410109.

[5] A. B. Balantekin and Y. Pehlivan, "Supersymmetry and Nuclear Pairing," J. Phys. G. 34 (2007) 1783-1788, arXiv:0705.1318 [nucl-th]. 
[6] L. E. Gendenshtein, "Derivation of exact spectra of the Schrodinger equation by means of supersymmetry," JETP Lett. 38 (1983) 356-359.

[7] S. Odake and R. Sasaki, "Calogero-Sutherland-Moser Systems, Ruijsenaars-Schneidervan Diejen Systems and Orthogonal Polynomials," Prog. Theor. Phys. 114 (2005) 12451260, arXiv:hep-th/0512155.

[8] G. E. Andrews, R. Askey and R. Roy, "Special Functions," Encyclopedia of mathematics and its applications, Cambridge, (1999).

[9] R. Koekoek and R.F. Swarttouw, "The Askey-scheme of hypergeometric orthogonal polynomials and its q-analogue," arXiv:math.CA/9602214.

[10] F. Calogero, "Solution of the one-dimensional $N$-body problem with quadratic and/or inversely quadratic pair potentials," J. Math. Phys. 12 (1971) 419-436.

[11] B. Sutherland, "Exact results for a quantum many-body problem in one-dimension. II," Phys. Rev. A5 (1972) 1372-1376.

[12] S. Odake and R. Sasaki, "Unified Theory of Annihilation-Creation Operators for Solvable ('Discrete') Quantum Mechanics," J. Math. Phys. 47 (2006) 102102 (33pages), arXiv:quant-ph/0605215.

[13] A. Degasperis and S. N. M. Ruijsenaars, "Newton-Equivalent Hamiltonians for the Harmonic Oscillator," Ann. of Phys. 293 (2001) 92-109.

[14] M. J. Morris, "Interpolation theory for quantum mechanics," J. Phys. A 5 (1972) 489494. 